\title{
Les coordinations de termes dissemblables sont-elles elliptiques ?*
}

\author{
François Mouret \\ CNRS, Laboratoire de Linguistique Formelle, \\ UMR 7110 CNRS \& Université Paris 7 \\ francois.mouret@linguist.jussieu.fr
}

\section{Le problème}

C'est un fait bien connu que les termes d'une coordination peuvent différer par divers aspects syntaxiques tels que la catégorie, le mode verbal ou encore la forme des prépositions introductrices dans certains environnements (1) tandis qu'ils doivent être strictement identiques concernant ces mêmes aspects ailleurs (2). Une généralisation descriptive simple, généralement attribuée à Thomas Wasow (cf. Gazdar et al. 1985 : 179, Pullum \& Zwicky 1986 : 752), rend immédiatement compte des distributions observées ${ }^{1}$ : les coordinations de termes dissemblables ne sont possibles que lorsque chacun des termes conjoints peut apparaître seul en lieu et place de la structure dans son ensemble à interprétation égale du matériel environnant. C'est le cas en (1), comme le montrent les données en (3), mais non en (2), comme le montrent les données en (4).

(1) a. Marie adorait [le cinéma] et [faire les boutiques]. (SN + SVinf)

b. Il semble [que Pierre ne viendra pas] et [que Marie soit en retard]. (que $\mathrm{Ph}[$ ind] + que $\mathrm{Ph}$ [subj])

c. Il était apprécié [de ses collègues] et [par tous ses étudiants]. (de $\mathrm{SN}+$ par $\mathrm{SN})$

(2) a. *Marie appréciait [le cinéma] et [faire les boutiques].

b. *Il s'avère [que Pierre ne viendra pas] et [que Marie soit en retard].

c. *Il était critiqué [de ses collègues] et [par tous ses étudiants].

(3) a. Marie adorait $\{[$ le cinéma] / [faire les boutiques]\}.

b. Il semble \{[que Pierre ne viendra pas] / [que Marie soit en retard]\}.

c. Il était apprécié $\{[$ de ses collègues] / [par tous ses étudiants]\}.

(4) a. Marie appréciait $\{[$ le cinéma $]$ / [faire les boutiques $]\}$.

b. Il s'avère $\{$ [que Pierre ne viendra pas] / *[que Marie soit en retard] $\}$.

c. Il était critiqué $\{*$ de ses collègues] / [par tous ses étudiants]\}.

Plus délicate en revanche est la question rebattue de l'intégration de cette généralisation dans le dispositif grammatical. Une première approche, initiée dans les années quatre-vingt en GPSG (Sag et al. 1985, Gazdar et al. 1985) et développée plus tard dans divers cadres (voir notamment Bayer \& Johnson 1995 en grammaire catégorielle et Sag 2002, 2005 en $\mathrm{HPSG}^{2}$ ), consiste à exploiter la représentation des catégories sous la forme de structures de traits. Sans rentrer ici dans le détail des propositions formelles, on retiendra que l'analyse met en avant les données de l'observation : on considère que la règle de coordination peut affecter aussi bien des termes syntaxiquement identiques que des termes dissemblables, les distributions observées résultant de la compatibilité ou non entre les traits de la coordination dans son ensemble, qui peuvent être sous-spécifiés lorsque les termes conjoints ont des propriétés conflictuelles, et les traits requis par l'environnement, qui peuvent être sous-spécifiés, comme en (1), ou non, comme en (2), et de fait constituer ou non un environnement approprié pour une coordination de termes dissemblables.

Alternativement, on a proposé de dériver les coordinations de termes dissemblables d'un mécanisme plus général d'ellipse (voir notamment van Oirsouw 1987, Wilder 1997 dans le cadre Principes et Paramètres 
et Crysmann 2003, Beavers \& Sag 2004, Chaves 2006 pour des propositions récentes en HPSG). Il existe en effet à côté des coordinations de constituants une classe de coordinations de non-constituants que nous appelons ici coordinations de séquences, qui se caractérisent en première approximation par la combinaison de deux séquences ou plus, composées de compléments (5a), d'ajouts (5b), ou d'un mélange des deux $(5 \mathrm{c})$ à droite d'un prédicat. Or il est souvent admis que ces tours mettent en jeu une règle d'ellipse sur la frontière gauche du second conjoint, comme l'illustrent les représentations informelles en (6). Admettons cette analyse. Il est clair que la règle en jeu permet également, quelle que soit sa formulation précise, de générer les diverses coordinations de catégories dissemblables et leur distribution particulière, comme l'illustrent les représentations en (7). On peut donc maintenir dans cette perspective que la règle de coordination n'affecte que des catégories syntaxiques identiques, les distorsions observées résultant de la possibilité (7a) ou non (7b) de reconstruire le matériel manquant sur la frontière gauche du second conjoint.

(5) a. Paul offrira < un disque à Marie $>$ et $<$ un livre à Jean $>$.

b. Paul joue au tennis $<$ le lundi avec Marie $>$ et $<$ le vendredi avec Jean $>$.

c. Paul joue $<$ au tennis le lundi $>$ et $<$ au football le mardi $>$.

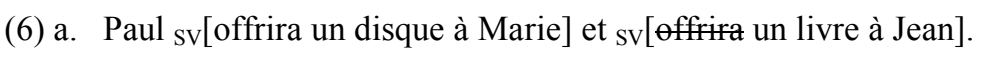

b. Paul sv[joue au tennis le lundi avec Marie] et sv[joue au tennis le vendredi avec Jean].

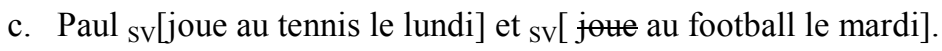

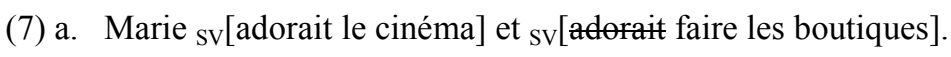

b. Marie ${ }_{\mathrm{sv}}$ [appréciait le cinéma] et $\left.\mathrm{Sv}^{*}\right)$ [appréciait faire les boutiques].

Dans cet article, nous montrons sur la base d'arguments empiriques relativement indépendants d'un cadre particulier qu'une analyse à base d'ellipse des coordinations de séquences doit être abandonnée : il faut admettre à l'instar des propositions en grammaire catégorielle (cf. notamment Dowty 1988, Steedman 1989, 1996, 2000) qu'on peut coordonner aussi bien des constituants que des séquences de constituants dans la portée syntaxique d'un prédicat. Il s'ensuit qu'un mécanisme d'ellipse sur la frontière gauche du second conjoint ne peut pas être exploité pour rendre compte des coordinations de termes dissemblables et de leur distribution particulière. Ce résultat est important à au moins deux titres. Il confirme d'abord, si besoin est, qu'une grammaire adéquate ne peut se dispenser d'une théorie élaborée des traits et de leur mode de propagation. Il relativise par ailleurs les approches syntagmatiques classiques qui s'appuient sur une organisation rigide en constituants.

\section{Propriétés syntaxiques des coordinations de séquences}

Les propriétés syntaxiques majeures des coordinations de séquences ont été bien identifiées (voir Hudson 1976, Dowty 1988 pour l'anglais, Abeillé \& Godard 2002 pour le français). Nous faisons le point sur les généralisations concernant le français dans cette première section.

\subsection{Propriétés générales}

Les coordinations de séquences présentent, comme nous le montrons, les propriétés générales des constructions coordonnées : elles peuvent mettre en jeu des conjonctions variées et obéissent aux mêmes contraintes de parallélisme que les coordinations de constituants ordinaires.

\subsubsection{Distribution des conjonctions}

Notons tout d'abord que les coordinations de séquences sont compatibles avec des conjonctions diverses (8), y compris les conjonctions doubles (9). On peut ajouter que l'absence de conjonction est également possible, comme l'illustrent les exemples en (10). 
(8) a. Il a offert un disque à Marie et un livre à Jean.

b. Il compte aller au théâtre avec Marie ou bien au cinéma tout seul.

c. Il ne joue plus au tennis le lundi ni au football le mardi.

d. Elle a donné de nombreux conseils à Jean mais aucun à Pierre.

e. Elle a bu un thé à dix heures ainsi qu'un café à midi.

(9) a. Il a offert et un disque à Marie et un livre à Jean.

b. Il compte aller \{ou bien / soit\} au théâtre avec Marie \{ou bien / soit $\}$ au cinéma tout seul.

c. Il ne joue plus ni au tennis le lundi ni au football le mardi.

(10) a. Il allait au cinéma avec Marie, au théâtre avec Jean, à l'opéra avec Pierre.

b. [A la réunion puis à Mayotte] [pour le premier], [au Mali puis au Benin] [pour le second], Dominique de Villepin et Nicolas Sarkozy se tiennent informés en temps réel des développements de l'affaire tout en feignant un relatif détachement. (Le Monde, 21/05/06)

\subsubsection{Contraintes de parallélisme}

Comme l'ont noté Abeillé \& Godard (2002), les coordinations de séquences respectent par ailleurs les contraintes de parallélisme couvertes par la généralisation de Wasow comme les coordinations de constituants ordinaires : chacune des séquences conjointes doit pouvoir apparaître seule en lieu et place de la coordination dans son ensemble. Il s'ensuit trois conséquences. Premièrement, les catégories de la séquence à droite de la conjonction peuvent partiellement différer des catégories à gauche à condition que chacune de ces catégories constitue un dépendant possible du prédicat en facteur (cf. (11)).

(11) a. Les enseignants attendent [des élèves] [qu'ils respectent les règles de l'établissement] et [de leur proviseur] [un soutien sans faille]. (SP-Ph[que] $+\mathrm{SP}-\mathrm{SN})$

$\mathrm{a}^{\prime}$. Les enseignants attendent \{[des élèves] [qu'ils respectent les règles de l'établissement] / [de leur proviseur] [un soutien sans faille]\}.

b. *Les enseignants estiment [des élèves] [qu'ils sont sérieux] et [de leur proviseur] [un homme sur lequel on peut compter]. $(* \mathrm{SP}-\mathrm{Ph}[$ que] $+\mathrm{SP}-\mathrm{SN})$

b'. Les enseignants estiment $\{$ [des élèves] [qu'ils sont sérieux] / *[de leur proviseur] [un homme sur lequel on peut compter]\}.

Deuxièmement, la séquence à droite de la conjonction peut comporter un nombre de dépendants différent du nombre de dépendants réalisés à gauche, et cela que ces dépendants correspondent à des compléments (12a), ou à des ajouts (12b). Notons que la phrase (12a) n'implique pas obligatoirement que c'est à sa mère (ou à quelqu'un d'autre) que Paul a écrit un petit poème, pas plus que la phrase (12b) n'implique que c'est avec Marie (ou avec quelqu'un d'autre) que Paul joue du piano le vendredi. Les coordinations de séquences n'observent donc pas, contrairement à ce qui est souvent admis, des contraintes de parallélisme syntaxique et sémantique plus fortes que les coordinations de constituants ordinaires ${ }^{3}$.

(12) a. Paul écrira [une lettre] [à sa mère] et [un petit poème]. (Abeillé \& Godard $2002: 414$ )

$$
(\mathrm{SN}-\mathrm{SP}+\mathrm{SN})
$$

b. Paul joue du piano [le lundi] [avec Marie] et [le vendredi]. (SN-SP $+\mathrm{SN})$

Enfin, comme les coordinations ordinaires, les coordinations de séquences respectent la contrainte d'extraction / cliticisation parallèle de Ross (1967) : l'extraction ou la cliticisation d'un constituant hors d'un conjoint n'est possible qu'à la condition d'opérer simultanément dans tous les autres conjoints (cf. (13)).

(13) a. une thèse dont il a lu [le dernier chapitre _ ] [hier] et [la conclusion _ ] [ce matin] 
b. *une thèse dont il a lu [le dernier chapitre _ ] [hier] et [votre article] [ce matin]

c. Votre thèse, Paul en a lu [le dernier chapitre _ ] [hier] et [la conclusion _ ] [ce matin].

d. *Votre thèse, Paul en a lu [le dernier chapitre _ ] [hier] et [votre article] [ce matin].

\subsection{Propriétés particulières}

Deux propriétés syntaxiques caractérisent spécifiquement les coordinations de séquences par rapport aux coordinations de constituants ordinaires. La première est bien connue : les coordinations de séquences échappent aux tests de constituance usuels tels que l'extraction, tout comme échappent aux tests de constituance les séquences non coordonnées ${ }^{4}$ :

(14) a. *Ce que Paul a offert, c'est un disque à Marie et un livre à Jean.

b. *Ce que Paul a offert, c'est un disque à Marie.

Par ailleurs, seules les coordinations de séquences de constituants de même niveau, c'est-à-dire dépendants d'un même prédicat, sont autorisées, comme le montre l'impossibilité de conjoindre par exemple des séquences composées d'un complément du nom et d'un complément du verbe (comparer (15a) et (15b)) (cf. Abeillé \& Godard 2002) .

(15) a. Paul a donné [les jouets de sa fille] [à Marie] et [les jouets de son fils] [à Pierre].

b. ??Paul a donné les jouets [de sa fille] [à Marie] et [de son fils] [à Pierre].

On pourrait penser qu'il s'agit là d'une contrainte analogue à celle qui est observée par les constructions "trouées" connues sous le nom de gapping dans la littérature anglo-saxonne, où seuls des constituants "majeurs" au sens d'Hankamer (1971), c'est-à-dire des dépendants du verbe principal ou d'un verbe enchâssé sont légitimes dans la séquence à droite de la conjonction (comparer (16a) et (16b)).

(16) a. Paul a donné les jouets de sa fille et [Jean] [les jouets de son fils].

b. ??Paul a donné les jouets de sa fille et [Jean] [de son fils].

Cette généralisation est inadéquate en ce qui concerne les coordinations de séquences. Elle ne permet pas en effet d'expliquer le fait que les coordinations de séquences sont autorisées dans les mêmes conditions dans le domaine nominal (17) ou adjectival (18), où la notion de constituant "majeur" ne fait pas sens ${ }^{6}$.

(17) a. Paul désapprouve les propositions [du ministre de l'économie] [en faveur de l'emploi] et [du ministre de l'éducation] [en faveur de la recherche].

b. *Paul désapprouve les propositions du ministre [de l'économie] [en faveur de l'emploi] et [de l'éducation] [en faveur de la recherche].

(18) a. Les résultats sont inférieurs [à la moyenne régionale] [de 15 pourcent] et [à la [moyenne nationale] [de 20 pourcent].

b. *Les résultats sont inférieurs à la moyenne [régionale] [de 15 pourcent] et [nationale] [de 20 pourcent].

Il faut conclure que les coordinations de séquences n'obéissent pas exactement aux mêmes contraintes que les constructions trouées et qu'on ne peut donc assimiler les unes et les autres.

\section{Analyses proposées}

Trois structures syntaxiques ont été proposées pour rendre compte des propriétés particulières des coordinations de séquences : une structure à ellipse de type A (voir entre autres van Oirsouw 1987, Wilder 1997 dans une perspective transformationnelle, et Crysmann 2003, Beavers \& Sag 2004, Chaves 2006 en 
HPSG), une structure à ellipse de type B (cf. Sag et al. 1985) et une structure sans ellipse de type C (cf. Dowty 1988, Steedman 2000 en grammaire catégorielle, Hudson 1988 en grammaire de dépendance, Maxwell \& Manning 1996 en LFG et Mela \& Fouqueré 1996 en HPSG). Nous précisons brièvement le contenu analytique de ces différentes structures en faisant abstraction des modèles formels particuliers dans lesquels elles ont été adoptées.
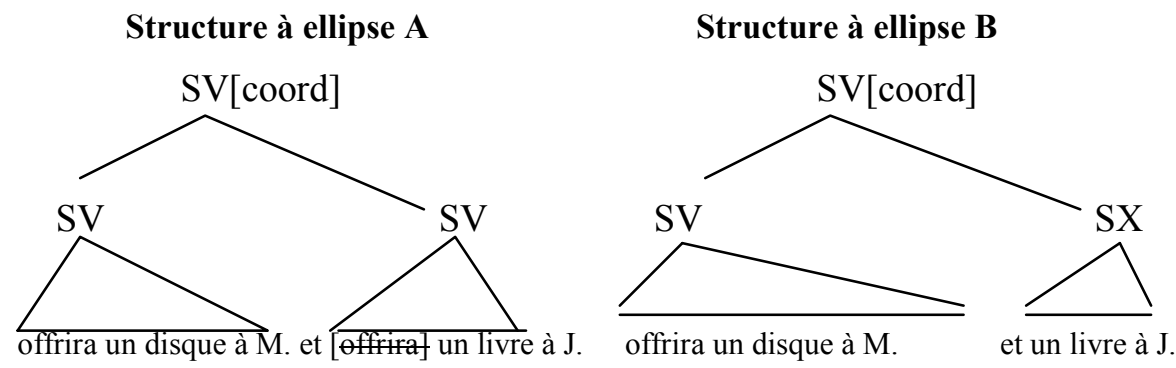

Structure sans ellipse C

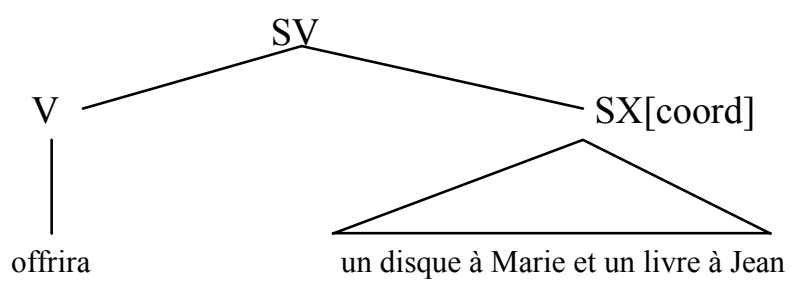

La structure A s'inscrit dans le cadre d'une approche syntaxique de l'ellipse. On postule que le verbe du second conjoint est présent dans la structure (de sorte qu'aucune règle d'interprétation spécifique n'est nécessaire), mais qu'il peut être effacé, c'est-à-dire non réalisé phonologiquement, lorsqu'il existe dans le conjoint qui précède un verbe identique. On peut admettre que l'identité syntaxique et sémantique qui légitime l'effacement est partielle, mais crucialement, une forme linguistique doit pouvoir être restituée. C'est ce que l'on appelle communément le principe de récupérabilité de l'effacement (cf. Chomsky 1964). La structure A laisse ainsi à penser que la séquence à droite de la conjonction d'une part, et la coordination dans son ensemble d'autre part, se comportent comme des groupes verbaux ordinaires.

La structure $\mathrm{B}$ repose sur une conception plus sémantique de l'ellipse. La séquence à droite de la conjonction est analysée comme une structure fragmentaire sans tête verbale, dont la bonne formation est déterminée par un principe de substitution. Grossièrement, on doit pouvoir remplacer certains des constituants du premier conjoint par les constituants de la séquence elliptique et obtenir une structure résultante syntaxiquement et sémantiquement bien formée. Cette approche ne suppose pas de reconstruction syntaxique du verbe à droite de la conjonction. Elle suppose en revanche, tout comme une approche adoptant la structure A, que la coordination dans son ensemble se comporte comme un groupe verbal ordinaire.

Enfin, la structure $\mathrm{C}$ rejette tout simplement l'idée que les coordinations de séquences mettent en jeu une ellipse. On admet dans cette perspective qu'il est possible de coordonner deux séquences ou plus de constituants et que ce type de coordination peut satisfaire les exigences de sous-catégorisation d'un prédicat et être interprétée compositionnellement comme une suite de constituants ordinaires ${ }^{7}$. Comme nous allons le voir à présent, seule cette dernière analyse permet de rendre compte de façon adéquate des propriétés syntaxiques des coordinations de séquences en français.

\section{Falsification des analyses à base d'ellipse}

Le choix d'une approche sans ellipse des coordinations de séquences est généralement guidé par des considérations théoriques. Nous le motivons dans cette section sur une base strictement empirique. Nous commençons par rappeler et compléter (\$4.1) les arguments syntaxiques avancés par Abeillé \& Godard 
(1996, 2000) à l'encontre d'une approche elliptique des coordinations de séquences, avant d'en présenter de nouveaux fondés sur la distribution des adverbes et certains phénomènes d'accord en faveur d'une approche sans ellipse $(\$ 4.2)$.

\subsection{Les arguments d'Abeillé \& Godard (1996, 2000)}

Comme l'ont noté Abeillé \& Godard (1996), la reconstruction du matériel supposé effacé dans le second conjoint des coordinations de séquences ne donne pas toujours lieu à une phrase grammaticale. Par exemple, une forme telle que ainsi que, qui présente dans certains de ses emplois les propriétés d'un conjonction de coordination (cf. Sabio 2005), peut se combiner à une séquence de constituants (19a), alors même qu'elle est incompatible avec un groupe verbal fini ou une phrase racine ailleurs (19b,c).

(19) a. Paul apportera un disque à Marie ainsi qu'un livre à Jean.

b. *Paul écoute la radio [ainsi que [lit le journal]].

c. *Paul lit le journal [ainsi que [Marie écoute la radio]].

On peut construire le même type de contraste avec les adverbes non et non pas, qui sont réservés à la négation de constituant (voir Culicover \& Jackendoff 2005 pour des données similaires en anglais). Outre leur emploi en corrélation avec la conjonction mais qu'on laisse ici de côté, ces deux adverbes peuvent apparaître immédiatement à droite de la conjonction et introduire une séquence de constituants (20a), alors même qu'ils sont incompatibles avec un groupe verbal fini ou une phrase racine $(20 \mathrm{~b}, \mathrm{c})$.

(20) a. Paul apportera un disque à Marie et \{non / non pas \} un livre à Jean.

b. *Paul écoute la radio et [ $\{$ non / non pas $\}$ [lit le journal]].

c. *Il neige et [non pas [il pleut]].

Admettons que les coordinations de séquences mettent en jeu une structure à ellipse de type A. Il faut alors considérer que l'effacement du verbe fini est obligatoire dans le second conjoint en (19a) et (20a), ce qui va à l'encontre du principe de récupérabilité.

Considérons à présent une approche des coordinations de séquences recourant à une structure à ellipse de type B. Suivant cette analyse, la séquence à droite de la conjonction constitue une structure fragmentaire dépourvue de tête verbale. Elle n'est donc pas spécifiée pour le mode. On prédit dès lors correctement sa compatibilité avec des formes telles que ainsi que ou non pas. Comme le notent Abeillé \& Godard (2000), une telle analyse rencontre cependant une difficulté lorsque l'on prend en compte les coordinations à redoublement. Comme l'illustrent les contrastes en (21-22), la conjonction initiale de la coordination se place obligatoirement après le prédicat, qu'il s'agisse d'un verbe (21a) ou non (21b), et non devant celui-ci (22), comme cela est attendu si ce prédicat appartient au premier terme conjoint.

(21) a. Paul compte apporter et un disque à Marie et un livre à Jean.

b. Les résultats sont inférieurs et à la moyenne régionale de 15 pourcent et à la moyenne nationale de 20 pourcent.

(22) a. *Paul compte et apporter un disque à Marie et un livre à Jean.

b. *Les résultats sont et inférieurs à la moyenne régionale de 15 pourcent et à la moyenne nationale de 20 pourcent.

Comme le suggèrent Beavers \& Sag (2004), on pourrait essayer de maintenir que les coordinations de séquences constituent, malgré les apparences, des coordinations de groupes verbaux elliptiques en considérant que les conjonctions initiales marquent non pas la frontière gauche du premier conjoint, mais plutôt la frontière entre le matériel mis en facteur par l'opération d'ellipse, c'est-à-dire le verbe offrir en (21a) et l'adjectif inférieur en (21b), et le matériel non partagé par les conjoints, c'est-à-dire les séquences de compléments. Cette analyse suppose une règle de placement spécifique des conjonctions initiales après le 
prédicat tête du syntagme avec lequel elles se combinent lorsqu'un prédicat identique est omis dans le second conjoint. Cette règle est peu plausible. Elle ne permet pas par ailleurs d'expliquer le fait que les locuteurs qui rejettent les coordinations à redoublement de groupes verbaux ou de phrases racines finies introduites par et ou $n i$ du type (23-24) acceptent tous en revanche les coordinations de séquences de la forme (25) (cf. Mouret 20005). Il faudrait, pour en rendre compte dans le cadre d'une structure à ellipse, conditionner la combinaison des conjonctions et ou $n i$ initiales à un SV fini et leur placement après le verbe tête de ce SV à l'ellipse obligatoire du verbe dans le second conjoint.

(23) a. \%Paul et lit le journal et écoute la radio.

b. \%Paul ni ne lit le journal ni n'écoute la radio.

(24) a. \%Ce matin, et Paul a lu le journal et Marie a écouté la radio.

b. \%Ce matin, ni Paul n'a lu le journal, ni Marie n'a écouté la radio

(25) a. Paul apportera et un disque à Marie et un livre à Jean

b. Paul n'apportera ni un disque à Marie ni un livre à Jean

Cette stipulation n'a aucune plausibilité et contraste avec les généralisations naturelles que permet une analyse des coordinations de séquences sans ellipse recourant à une structure de type $\mathrm{C}$. Admettons en effet une telle structure. Suivant cette analyse, le prédicat verbal est extérieur à la structure coordonnée. On prédit dès lors correctement le placement de la conjonction initiale des coordinations à redoublement devant la première séquence, et non devant le prédicat partagé (cf. 26), ainsi que le caractère non fini du second conjoint (27), comme du premier (28), étant admis que ni l'une ni l'autre des séquences conjointes ne comporte de verbe.

(26) a. Paul compte apporter et un disque à Marie et un livre à Jean.

b. *Paul compte et apporter un disque à Marie et un livre à Jean

(27) a. Paul apportera un disque à Marie ainsi qu' un livre à Jean.

b. Paul apportera un disque à Marie et non pas un livre à Jean.

(28) a. Paul apportera et un disque à Marie et un livre à Jean.

b. Paul n'offrira ni un disque à Marie ni un livre à Jean.

\subsection{Autres arguments}

Nous présentons deux arguments empiriques supplémentaires qui militent contre une approche à base d'ellipse des coordinations de séquences, et corrélativement en faveur de la structure C. Le premier repose sur la distribution et l'interprétation de certains adverbes, et le second sur les phénomènes d'accord dans les tours à sujet inversé.

\subsubsection{Distribution et interprétation des adverbes}

Considérons d'abord les adverbes restrictifs et additifs. Comme le montrent les données en (29), ces adverbes peuvent introduire une coordination de séquences et s'y "associer" sémantiquement. Grossièrement, l'adverbe seulement en (29a) dit que parmi l'ensemble des paires $<\mathrm{x}, \mathrm{y}>$ telles que Paul offre x à y à un moment $t$ ' après le moment présent $t 0$, seule la conjonction des paires $<$ un disque, Pierre $>$ et $<$ un livre, Marie> donne lieu à une proposition vraie lorsqu'elle est combinée au prédicat. En (29b), l'adverbe additif aussi dit à l'inverse que parmi l'ensemble des paires $<\mathrm{x}, \mathrm{y}>$ telles que Paul offre $\mathrm{x}$ à y à un moment $\mathrm{t}$ ' après le moment présent t 0 , il existe une paire autre que la conjonction des paires $<$ un disque, Pierre $>$ et $<$ un livre, Marie> qui donne lieu à une proposition vraie lorsqu'elle est combinée au prédicat. 
(29) a. Paul offrira seulement $<$ un disque à Pierre et un livre à Marie> alors qu'il aurait pu offrir aussi une bouteille de vin à Jean.

b. Paul offrira aussi <un disque à Pierre et un livre à Marie> alors qu'il aurait pu offrir seulement une bouteille de vin à Jean.

Ces phénomènes d'association sont problématiques pour les approches fondées sur une structure à ellipse de type A ou de type B. On ne voit pas en effet comment l'adverbe peut prendre la coordination dans son ensemble comme associé sémantique s'il est enchâssé dans le premier terme conjoint. Une telle association hors du premier terme conjoint est en effet exclue dans les coordinations ordinaires de syntagmes verbaux, comme le montre l'absence d'équivalence entre (30a) et (30b) d'une part, et entre (30c) et (30d) d'autre part.

(30) a. Paul compte [[lire seulement le journal] [et écouter la radio]].

b. $\measuredangle=/=>$ Lire le journal et écouter la radio sont les seules choses que Paul compte faire.

c. Paul compte [[lire aussi le journal] [et écouter la radio]]

d. $\langle=/ \Rightarrow>$ Outre lire le journal et écouter la radio, il y a autre chose que Paul compte faire.

Une solution potentielle consisterait à disjoindre partiellement le domaine d'ordre du domaine de constituance, suivant en cela les propositions de Reape (1994) et Kathol (2000). On pourrait par exemple considérer que l'adverbe en (29a) ou (29b) s'adjoint, malgré les apparences, à la coordination elliptique de groupes verbaux ou à la phrase dans son ensemble, sa linéarisation parmi les constituants du premier conjoint résultant d'une règle d'ordre indépendante. Cette solution est clairement inadéquate. Comme le montrent les données en (31), l'adjonction d'un adverbe restrictif ou additif à un groupe verbal fini ou à une phrase racine est en effet exclue de façon indépendante en français ${ }^{8}$.

(31) a. *Paul [seulement [lit le journal]] alors qu'il pourrait aussi écouter la radio.

b. *[Seulement [il pleut]] alors qu'il pourrait aussi faire froid.

c. *Paul [aussi [lit le journal]] alors qu'il pourrait seulement écouter la radio.

d. *[Aussi [il pleut]] alors qu'il pourrait seulement faire froid.

D'un autre côté, une approche sans ellipse des coordinations de séquences ne rencontre aucune de ces difficultés. On peut admettre dans cette perspective que l'adverbe s'adjoint à la coordination de séquences dans son ensemble ou bien qu'il est réalisé au même niveau que celle-ci dans une structure plus plate. Dans les deux cas, l'adverbe a accès localement au contenu de la coordination requis pour construire l'interprétation restrictive ou additive.

\subsubsection{Inversion du sujet et phénomènes d'accord}

Les phénomènes d'accord dans les tours à inversion du SN sujet fournissent un argument supplémentaire en faveur d'une analyse sans ellipse des coordinations de séquences. Nous nous limiterons ici au cas des SN inversés dans les tours narratifs (cf. Marandin 1999)':

(32) [Tout était calme].

a. Alors surgit d'un champ un renard.

b. Alors surgirent d'un champ deux renards.

On peut coordonner des séquences comportant chacune un sujet postverbal et un ou plusieurs compléments. On observe dès lors deux stratégies d'accord possibles. Le verbe doit pouvoir s'accorder indépendamment avec chacun des sujets postverbaux lorsque la coordination est interprétée comme une conjonction de deux événements successifs, comme l'illustrent les données en (33), où la relation de succession est imposée par l'adverbial quelques secondes plus tard. Le verbe apparaît en revanche obligatoirement au 
pluriel, et ce quelle que soit la valeur de nombre de chacun des sujets postverbaux, lorsqu'une relation symétrique est mise en jeu, comme l'illustrent les données en (34), où la relation en question est renforcée par la présence de l'adverbe simultanément.

(33) a. Alors \{surgit / *surgirent\} d'un champ un renard et quelques secondes plus tard d'un buisson une biche.

b. ??Alors surgirent d'un champ deux renards et quelques secondes plus tard d'un buisson une biche.

(34) a. Alors \{*surgit / surgirent $\}$ simultanément d'un champ un renard et d'un buisson une biche.

b. Alors surgirent simultanément d'un champ deux renards et d'un buisson une biche.

La première stratégie d'accord est compatible avec une structure à ellipse de type $\mathrm{A}$ ou de type $\mathrm{B}$. La deuxième stratégie est en revanche problématique. On voit mal en effet comment expliquer la forme plurielle du verbe en (34a) si celui-ci appartient au premier conjoint ${ }^{10}:$ il faudrait alors admettre que l'ellipse dans le second conjoint rend licite dans le premier la combinaison par ailleurs exclue d'un verbe au pluriel avec un sujet singulier, une stipulation peu attrayante ${ }^{11}$.

Il est clair en revanche que ni l'une ni l'autre des stratégies d'accord observées n'est exclue a priori si l'on adopte une structure sans ellipse de type C. Suivant cette analyse, le verbe est extérieur à la structure coordonnée, ce qui laisse donc ouverte la possibilité de formuler des contraintes d'accord spécifiques entre ce verbe et la coordination de séquences dans son ensemble.

\subsubsection{Synthèse}

Dans cette section, nous avons présenté une falsification détaillée des analyses recourant à une règle d'ellipse pour rendre compte des coordinations de séquences. Comme nous l'avons montré, ni une approche fondée sur l'effacement, ni une approche fondée sur une règle de légitimation des fragments à l'interface syntaxe-sémantique ne permet de représenter adéquatement les propriétés syntaxiques des coordinations de séquences du français. Nous concluons que ces tours ne mettent en jeu aucune ellipse : une grammaire adéquate doit ainsi autoriser non seulement les coordinations de constituants ordinaires, mais aussi les coordinations de séquences de constituants dans la portée syntaxique du prédicat. Ce résultat est en accord avec les hypothèses de la grammaire catégorielle (cf. Dowty 1988, Steedman 1989, 1996, 2000). Il est également en accord avec les hypothèses avancées dans divers cadres surfacistes (cf. notamment Hudson 1988 en grammaire de dépendance, Mela \& Fouqueré 1996 en HPSG). Nous y parvenons ici sur une base empirique. Nous explicitons dans la conclusion qui suit les conséquences de ce résultat sur l'analyse des coordinations de termes dissemblables.

\section{Conclusion}

Revenons au problème posé en introduction. Nous avons vu qu'il existe deux approches possibles du degré d'identité des termes conjoints dans les coordinations. La première consiste à autoriser non seulement les coordinations de termes identiques, mais aussi les coordinations de termes dissemblables et à recourir à une théorie des traits pour rendre compte des distributions observées. La seconde consiste à dériver la distribution des coordinations de termes à première vue dissemblables d'une théorie plus générale de l'ellipse comme illustré en (35).

(35) a. Marie sv[adorait le cinéma] et sv[adorait faire les boutiques].

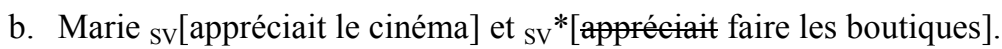

Une approche du degré d'identité des termes conjoints fondée sur l'ellipse est motivée par l'hypothèse que la règle en question est requise de façon indépendante pour caractériser les coordinations de séquences, ainsi conçues comme des coordinations réduites de la forme (36). 
(36) a. Paul ${ }_{\text {sv}}[$ offrira un disque à Marie] et sv[өffrira un livre à Jean].

b. Paul sv[joue au tennis le lundi avec Marie] et sv[joue autennis le vendredi avec Jean].

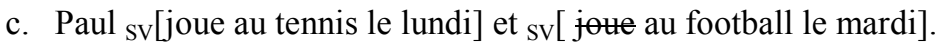

Or comme nous l'avons montré en détail dans la section précédente, cette dernière hypothèse est empiriquement inadéquate : les coordinations de séquences ne peuvent pas être analysées comme des structures elliptiques. Comme on le vérifie aisément, les divers arguments avancés s'appliquent également aux coordinations de termes dissemblables. En particulier, les coordinations de termes dissemblables sont compatibles avec des formes telles que ainsi que ou non pas (37) et peuvent être introduites par une conjonction initiale telle que et ou $n i$ dans le cadre d'une structure à redoublement (38), ou par un adverbe restrictif ou additif dont elles constituent l'associé sémantique (39).

(37) a. Marie adore le cinéma ainsi que faire les boutiques.

b. Marie adore le cinéma et non pas faire les boutiques.

(38) a. Marie adore et le cinéma et faire les boutiques.

b. Marie n'adore ni le cinéma ni faire les boutiques.

(39) a. Marie adore aussi le cinéma et faire les boutiques.

b. Marie adore seulement le cinéma et faire les boutiques.

Comme nous l'avons vu, ces diverses combinatoires invalident les analyses à base d'ellipse. Nous en tirons à présent la conclusion qui s'impose : les coordinations de termes dissemblables doivent être analysées telles quelles, sans recourir à un mécanisme d'ellipse. En d'autres termes, une théorie des traits et de leur mode de propagation dans les structures coordonnées est requise pour rendre compte du problème de l'identité relative des termes conjoints. Cette proposition est somme toute assez commune dans le paysage théorique contemporain. Nous espérons l'avoir motivée dans cet article sur une base empirique qui s'affranchit au moins partiellement des considérations théoriques, voir esthétiques, sur la forme que doit revêtir une grammaire.

\section{Bibliographie}

Abeillé, A. and D. Godard. 1996. La complémentation des auxiliaires en français. Langages 122, 32-61.

Abeillé, A. and D. Godard. 2000. Varieties of esse in Romance Languages. In D. Flinckinger et al. (eds), Berkeley Formal Grammar Conference. Stanford: CSLI Publications.

Abeillé, A. and D. Godard. 2002. The syntactic structure of French auxiliaries. Language, 78. 404-452.

Bayer, S. et M. Johnson. 1995. Features and agreement. Proceedings of ACL95, 70-76.

Beavers, J. and I. A. Sag. 2004. Coordinate ellipsis and apparent non-constituent coordination. In S. Müller (ed), Proceedings of the HPSG04 Conference. Stanford: CSLI on-line Publications.

Bonami, O., D. Godard et J.-M. Marandin. 1999. Constituency and word order in ... French Subject Inversion in Extraction Contexts.In G. Bouma, E. Hinrichs et G.-J. Kruijff (eds.), 'Constraints and ressources in natural language syntax and semantics'. Stanford : CSLI Publications.

Chaves, R. 2006. Coordination of unlikes without unlike categories. In S. Müller (ed), Proceedings of the HPSG06 Conference. Stanford : CSLI on-line Publications.

Chomsky, N. 1964. Current issues in linguistic theory. The Hague: Mouton.

Crysmann, B. 2003. An asymmetric theory of peripheral sharing in HPSG : conjunction reduction and coordination of unlikes. In Jager et al. (eds), Formal Grammar Proceedings 2003.

Culicover, P. and R. Jackendoff. 2006. Simpler syntax. Oxford University Press.

Dalrymple, M. et R. M. Kaplan. 2000. Feature indeterminacy and feature resolution. Language 76, 759-798. 
Dowty, D. 1988. Type raising, functional composition, and non-constituent conjunction. In R. Oehrle, E. Bach, et D. Wheeler (eds), Categorial Grammar and Natural Language Structures. Dordrecht: Kluwer. 153-198.

Guillet, A. et C. Leclère 1981. Restructuration du groupe nominal, Langages 63, 99-125.

Hankamer, J. 1971. Constraints on deletion in syntax. Thèse de $\mathrm{PhD}$, Université de Yale.

Hudson, R. 1988. Coordination and grammatical relations. Journal of Linguistics 24. 303-342.

Kaplan, R. M. et J. T. III Maxwell. 1988. Constituent coordination in lexical functional grammar. In Proceedings of COLING88, 303-305.

Kathol, A. 2000. Linear syntax. Oxford : Oxford University Press.

Larson, R. 1988. On the double object construction. Linguistic Inquiry, 19. 335- 391.

Larson, R. 1990. Double objects revisited: reply to Jackendoff. Linguistic Inquiry 21: 589-632

Leclère, C. 1978. Sur une classe de verbes datifs, Langue francaise 39, 66-75.

Marandin, J.-M. 1999. French unaccusative inversion. in Y. d'Hulst et al. (eds), Romance Languages and Linguistic Theory. Amsterdam: John Benjamins.

Maxwell, J. T. and C. Manning. 1996. A Theory of non-constituent coordination based on finite-state rules. In Butt, M. and T. King (eds), Proceedings of the first LFG Conference. Stanford: CSLI on-line Publications.

Mela, A. and C. Fouqueré. 1996. Coordination as a direct process. In Proceedings of ACL96. 124-130.

Mouret, F. 2005. La syntaxe des coordinations corrélatives. Langages 160, 67-92.

Mouret, F. 2006. A phrase structure approach to argument cluster coordination. In S. Müller (ed), Proceedings of HPSG 06. Stanford : CSLI on-line Publications.

Mouret, F. 2007. Grammaire des constructions coordonnées. Coordinations simples et coordinations à redoublement en français contemporain. Thèse de doctorat, Université Paris 7.

van Oirsouw, J. 1987. The syntax of coordination. London: Croom Helm.

Peterson, P. 2004. Coordination : consequences of a lexical functional account. Natural Language and Linguistic Theory 22-3, 649-699.

Pullum, G. et A. Zwicky. 1986. Phonological resolution of syntactic feature Conflicts. Language 62, 751- 773.

Reape, M. 1994. Domain union and word order variation in German. In J. Nerbonne, K. Netter et C. Pollard (eds), German head driven phrase structure grammar. Stanford : CSLI Publications. 151-197.

Ross, J. 1967. Constraints on variables in syntax. thèse de Phd, MIT.

Sabio, F. 2005. Sur la syntaxe des paradigmes : les listes en ainsi que et de même que. Poster présenté au colloque Typologie et modélisation de la coordination et de la subordination, Université Paris 3, 26-28 mai 2005.

Sag, I. A. 2002. Coordination and underspecification. In J.-B. Kim and S. Wechsler (eds), Proceedings of HPSG 02 Conference. CSLI on-line Publications.

Sag, I. A. 2005. La coordination et l'identité syntaxique des termes. Langages 160, 110-128.

Sag, I. G. Gazdar, T. Wasow, and S. Weisler. 1985. Coordination and how to distinguish categories. Natural Language and Linguistic Theory, 3.2. 117-172.

Schachter, P. and S. Mordechai. 1983. A phrase structure account of non-constituent conjunction. In Proceedings of the 2nd West Coast Conference on Formal Linguistics. Stanford : StanfordLinguistics Association. 260-274.

Steedman, M. 1989. Constituency and coordination in combinatory grammar. In M. Baltin et A. Kroch (eds), Alternative conceptions of phrase structure. Chicago : the University of Chicago Press. 201-231.

Steedman, M. 1996. Surface structure and interpretation. Cambridge : MIT Press.

Steedman, M. 2000. The syntactic process. Cambridge: MIT Press.

Wilder, C. 1997. Some properties of ellipsis in coordination. In A. Alexiadou and T. Hall (eds), Studies on Universal Grammar and Typological Variation. Amsterdam: John Benjamins. 59-107. 
Zaenen, A. et R. Kaplan. 2003. Subject inversion in French : equality and inequality in LFG. In C. Beyssade, O. Bonami, P. Cabredo Hofherr, and F. Corblin (eds), Empirical issues in syntax and semantics 4. Paris : Presses Universitaires de Paris-Sorbonne. 190-205.

* La présente étude a été financée dans le cadre du projet PRO-GRAM soutenu par l'ANR. Nous laissons de côté ici les questions de modélisation. Pour des propositions d'analyse en HPSG, voir Mouret (2006, 2007).

${ }^{1}$ Les données de l'accord échappent à première vue à cette généralisation (cf. (i-ii)). Voir Mouret (2007 : 37-41) pour une discussion.

(i) [Paul] et [Marie] partent demain.

(ii) $*\{[$ Paul $] /[$ Marie $]\}$ partent demain.

${ }^{2}$ Les travaux en LFG illustrent une position intermédiaire avec un traitement elliptique des coordinations de catégories différentes (Maxwell \& Manning 1996) et des règles de distribution des traits spécifiques pour les coordinations de termes différant sur des propriétés telles que le mode verbal, la forme des prépositions, etc. (cf. Kaplan \& Maxwell 1988, Dalrymple \& Kaplan 2000, Peterson 2004).

${ }^{3}$ Les verbes qui acceptent sans altération de leur identité lexicale deux compléments ou un seul et de fait des coordinations de séquences de longueurs différentes sont relativement peu nombreux. Outre les verbes susceptibles de fonctionner comme tête d'un prédicat complexe étudiés notamment par Abeillé \& Godard (2002), on peut citer les verbes à complément datif étendu (Leclère 1978) tels que écrire, fabriquer, construire, etc. et ceux qui autorisent "une restructuration du SN" (Guillet \& Leclère 1981) :

(i) Ce texte a heurté (et) [la sensibilité de Marie] et [Pierre] [dans ses convictions].

(ii) Ce texte a provoqué (et) [l'enthousiasme de Pierre] et [de la stupeur] [chez Marie].

(iii) Paul a acheté (et) [des litres de bière] et [du pain] [par kilos].

${ }^{4}$ Ces exemples sont acceptables lorsque la séquence extraite est employée comme citation dans les contextes de reprise. On vérifie que les coordinations de séquences tout comme les séquences non coordonnées ne sont pas extractibles dans les tours qui n'autorisent pas ce type d'emploi, tels que les concessives :

(i) *Quelques disques à Marie et quelques livres à Jean qu'il offre, ils sont toujours contents.

(ii) *Quelques disques à Marie qu'il offre, elle est toujours contente.

${ }^{5}$ Un relecteur anonyme nous signale la possibilité d'améliorer l'acceptabilité de ce type d'exemples avec une prosodie appropriée. Une étude expérimentale reste à faire sur ce point.

${ }^{6}$ Les séquences composées d'un complément du verbe matrice et d'un complément d'un verbe enchâssé semblent moins réfractaires à la coordination (i), ce qui s'explique sans doute par la possibilité d'analyser la construction comme une coordination phrastique "trouée" de la forme (ii) plutôt qu'une coordination de séquences proprement dite.

(i) ?Paul a ordonné de traduire [Ovide] [à ses élèves latinistes] et [Euripide] [à ses élèves hellénistes].

(ii) [Paul a ordonné de traduire Ovide à ses élèves latinistes $]_{\mathrm{Ph}}$ et [[Euripide] [à ses élèves hellénistes] $]_{\mathrm{Ph}}$.

${ }^{7} \mathrm{Il}$ a été proposé que la structure $\mathrm{C}$ constitue un cas de "montée du nœud gauche" avec extraction parallèle du verbe hors de chacun des SV conjoints (cf. Schachter \& Mordechai 1983, Larson 1988, 1990). Comme noté par Dowty (1988), l'approche surfaciste de Schachter \& Mordechai ne rend pas compte des cas tels que (i-ii) où le matériel partagé par les séquences comprend non seulement le verbe tête, mais aussi un ou plusieurs dépendant(s), c'est-à-dire une suite qui ne correspond pas à un constituant. Elle ne rend pas non plus compte des problèmes de reconstruction soulevés ici même dans la section 4 . 
L'analyse dérivationnelle proposée par Larson, qui s'appuie sur une règle de mouvement du verbe lexical dans le domaine flexionnel, ne rencontre aucune de ces difficultés.

(i) Paul a apporté des fleurs <à Marie hier et à Léa ce matin>.

(ii) Paul joue au tennis <avec Marie le lundi et avec Paul le jeudi>.

${ }^{8}$ Les coordinations de séquences peuvent également constituer l'argument sémantique d'adverbes tels que respectivement, à la fois, tour à tour, qui présentent une distribution comparable :

(i) Paul offrira respectivement un disque à Marie et un livre à Jean.

(ii) Paul a présenté tour à tour Jean à Marie et Pierre à Jeanne.

(iii) Paul joue alternativement au football avec Pierre et au tennis avec Marie.

${ }^{9}$ Les données sont les mêmes en cas d'inversion stylistique dans les constructions à extraction, y compris dans les cas d'inversion longue identifiés par Bonami et al. (1999) où le sujet du verbe matrice apparaît parmi les compléments d'un verbe enchâssé (cf. (i-ii)). Ces données sont compatibles avec une analyse qui génère le sujet dans la position dans laquelle il apparaît linéairement (cf. Zaenen \& Kaplan 2003). Elles sont problématiques pour les approches qui disjoignent plus radicalement la combinatoire et la linéarisation. Admettons en effet que le sujet est combiné avec le verbe matrice et linéarisé parmi les dépendants enchâssés comme proposé par Bonami et al. (1999). Le sujet et les compléments enchâssés ne constituent pas dans cette perspective des dépendants de même niveau et l'on s'attend donc à ce que des coordinations de séquences constituées chacune d'un sujet postverbal et d'un complément enchâssé soient exclues contrairement aux faits observés.

(i) C'est une triste nouvelle qu' $\left\{{ }^{*}\right.$ ont $\left./ \mathrm{a}\right\}$ dû annoncer le conseiller d'éducation aux élèves, puis le proviseur aux parents.

(ii) C'est une triste nouvelle qu' $\{$ ont $/ * a\}$ simultanément dû annoncer le conseiller d'éducation aux élèves et le proviseur aux parents.

${ }^{1010}$ Les coordinations de séquences diffèrent de ce point de vue des constructions trouées où le verbe réalisé s'accorde toujours avec le sujet de la phrase dans laquelle il apparait :

(i) Paul \{a / *ont $\}$ mangé une pizza et les enfants des lasagnes.

${ }^{11}$ Une solution alternative, suggérée par Chaves (2006), consisterait à considérer que la phrase (34a) constitue, lorsque le verbe est au pluriel, une phrase "semi-grammaticale", c'est-à-dire une phrase agrammaticale légitimée par un processus d'intégration sémantique relevant de la performance. Les arguments empiriques en faveur d'une telle analyse font défaut. En particulier, l'acceptabilité des tours du type (34a) avec le verbe au pluriel n'est en aucun cas intermédiaire par rapport à celle des tours du type (33a) avec le verbe au singulier. 